\title{
Informationen der Arbeitsgemeinschaft Knochentumoren e. V. (agkt)
}

\section{(Stand 12/20)}

\section{Vorsitzender:}

Prof. Dr. med. Klaus Wörtler

Institut für diagnostische und interventionelle Radiologie

Technische Universität München Ismaninger Straße 22, D-81675 München

E-Mail: klaus.woertler@tum.de

\section{Stellv. Vorsitzender:}

Prof. Dr. med. Jendrik Hardes Abteilung für onkologische Chirurgie am Sarkomzentrum der Universitätsklinik Essen Hufelandstraße 55, D-45147 Essen

E-Mail Jendrik.Hardes@uk-essen.de

\section{Beisitzer:}

Prof. Dr. med. Thomas F.E. Barth Institut für Pathologie, M23 Universität Ulm Albert-Einstein-Allee 1, D-89081 Ulm Email: thomas.barth@uniklinik-ulm.de

\section{Kassenwart:}

Prof. Dr. med. Axel Hillmann

Abteilung für Sarkome und Muskuloskelettale Tumore

Klinik für Unfallchirurgie, Orthopädie und Sportmedizin

Krankenhaus Barmherzige Brüder

Regensburg

Prüfeninger Straße 86, D-93049 Regensburg E-Mail: Axel.Hillmann@barmherzige-regens burg.de

\section{Geschäftsführer:}

Prof. Dr. med. Gernot Jundt Knontchentumor-Referenzzentrum und DÖSAK-Referenzregister am Institut für Pathologie

Universitätsspital Basel

Schönbeinstr. 40, CH-4031 Basel

E-Mail: sekretariat@agkt.org

\section{Mitgliedsbeitrag}

Der Mitgliedsbeitrag für ordentliche Mitglieder beträgt pro Jahr für Fachärzte Euro 100,-, für Assistenten Euro 50,- (Deutschland, Österreich, Schweiz) bzw. Euro 50,-/ 25,- (Kroatien, Polen, Ungarn und Tschechien).

\section{agkt im Internet}

www.agkt.org

Die nächste Arbeitstagung findet voraussichtlich am 16./17.4.2021 in Dresden statt. Falls sich noch Veränderungen ergeben, konsultieren Sie bitte die Homepage der AGKT, ebenso für Tagungsanmeldungen - Gäste willkommen - und Einzelheiten zur Mitgliedschaft (www.agkt.org).

\section{IMPRESSUM}

Verantwortlich für den Inhalt

Prof. Dr. Daniel Baumhoer, Basel

Prof. Dr. Gernot Jundt, Basel 